\title{
ILCEA
}

Revue de l'Institut des langues et cultures

d'Europe, Amérique, Afrique, Asie et Australie

$9 \mid 2007$

L'implicite et les écrits de l'entreprise

\section{L'implicite dans deux campagnes de communication du New York Stock Exchange (1920-1970)}

Implicit Messages in Two Marketing Campaigns of the New York Stock

Exchange (1920-1970)

\section{Michel Van der Yeught}

\section{OpenEdition}

\section{Journals}

Édition électronique

URL : http://journals.openedition.org/ilcea/635

DOI : $10.4000 /$ ilcea.635

ISSN : 2101-0609

Éditeur

UGA Éditions/Université Grenoble Alpes

Édition imprimée

Date de publication : 15 décembre 2007

Pagination : 49-71

ISBN : 978-2-84310-104-5

ISSN : 1639-6073

Référence électronique

Michel Van der Yeught, «L'implicite dans deux campagnes de communication du New York Stock Exchange (1920-1970) », ILCEA [En ligne], 9 | 2007, mis en ligne le 09 juin 2010, consulté le 02 mai 2019. URL : http://journals.openedition.org/ilcea/635; DOI : 10.4000/ilcea.635 


\title{
L'implicite dans deux campagnes de communication du New York Stock Exchange (I920-I970)
}

\author{
Michel VAN DER YeUGHT \\ Université de Toulon
}

\begin{abstract}
RÉSUMÉ
Afin d'influencer le public, le monde des affaires use largement de l'implicite dans ses campagnes promotionnelles pour éviter de s'exposer aux critiques que pourraient lui valoir des messages explicites. Cet article étudie la part d'implicite contenue dans deux slogans diffusés par la Bourse de New York (New York Stock Exchange) dans le cadre de deux campagnes promotionnelles lancées dans les années 1920 et les années 1950. Il analyse les conditions de création des slogans, les présupposés et les logiques sous-jacentes aux messages. Il montre comment le recours à l'implicite vise à manipuler les destinataires et à les faire adhérer à une idéologie.
\end{abstract}

ABSTRACT

In order to influence the public, businessmen fill their marketing campaigns with implicit messages and tend to avoid explicit ones that may expose them to criticism. This article deals with the implicit content of two slogans published by the New York Stock Exchange in two promotional campaigns in the 1920s and in the 1950s. It analyzes the conditions leading to the creation of the slogans, the assumptions and the underlying logic of the messages. It shows how the use of implicit content helps manipulate the public into adhering to an ideology.

Mots-clés - Implicite, Bourse de New York, Wall Street, slogan, campagne, promotion.

Key words - Implicit messages, New York Stock Exchange, Wall Street, slogan, campaign, marketing. 
Cet article étudie la part d'implicite contenue dans deux slogans diffusés par la Bourse de New York (New York Stock Exchange ou NYSE) dans le cadre de deux campagnes promotionnelles lancées dans les années 20 et au cours de la période 1950-1970. Aux époques considérées, les messages envoyés par le NYSE au grand public sont soumis à de nombreuses contraintes opposées. La Bourse est en butte à l'hostilité de l'opinion mais doit éviter de se présenter en situation défensive. Elle lutte pour défendre le volume d'affaires de ses membres mais il lui faut présenter son activité comme bénéfique à tous les Américains. Elle s'efforce de préserver son indépendance face aux tentatives de contrôle du pouvoir fédéral mais elle ne peut se permettre de s'opposer de front à la légitimité démocratique du Congrès. Les slogans choisis doivent tenir compte de ces divers obstacles et il importe que leur contenu explicite ne prête pas le flanc à des interprétations négatives. Ils procèdent donc par insinuations, par sous-entendus, par inférences et par allusions, afin de dire tout en pouvant prétendre qu'ils n'ont pas dit. Paul Grice distingue l'explicite qui se propose de dire quelque chose («to tell something») de l'implicite qui vise à amener quelqu'un à penser quelque chose («to get someone to think something») (Kerbrat-Orecchioni 1986 : p. 8, 21). Nous suivons Grice dans sa démarche en posant comme hypothèse que dans les slogans du NYSE la part implicite est plus signifiante que la part explicite car c'est elle qui influence les destinataires des messages et qui les amène à changer d'avis. Grâce aux présupposés, aux sous-entendus et aux logiques enfouies dont ils sont porteurs, les contenus implicites révèlent les rapports de force et les enjeux sous-jacents aux messages. Ils usent d'approches indirectes et suggestives qui leur permettent de respecter les convenances sociales et politiques de l'époque tout en jouant sur les valeurs auxquelles les destinataires sont sensibles. Décoder ces contenus implicites permet de comprendre les stratégies de démonstration et d'évitement qui ont été mises en oeuvre pour amener les destinataires à adopter un certain point de vue. Cette étude s'inspire largement des travaux que Catherine Kerbrat-Orechionni présente dans son ouvrage L'implicite (1986) et elle fait appel à plusieurs des outils d'analyse proposés par l'auteur. La première partie de l'article étudie le slogan «The NYSE is the Free and Open People's Market» émis dans les années 20 et la deuxième est consacrée au slogan «Own Your Share of American Business» régulièrement utilisé à des fins promotionnelles dans les années 50 et 60 . Afin d'éclairer l'analyse, le décodage des contenus implicites des slogans est chaque fois précédé d'une présentation du contexte socio-historique où ils sont apparus. 


\section{L'implicite dans le slogan «The Free and Open People's Market» (1920-1929)}

\section{Le contexte de l'émission du message}

Le NYSE lança la première campagne promotionnelle de son histoire dans les années 20 pour dissiper l'hostilité du public accumulée pendant la période dite progressive (Progressive Era, 1900-1914). À l'époque, le NYSE est déjà la plus grande bourse de valeurs des États-Unis et il a activement participé au développement des transports et à l'expansion industrielle du pays. Il pâtit pourtant d'une mauvaise presse car la concentration financière des Money Interests de Wall Street inquiète l'opinion et suscite les critiques des muckrakers contre les grandes banques et la Bourse. À la suite des krachs de 1893 et de 1907, en partie causés par la spéculation, les investigations officielles se sont multipliées et ont exposé sur la place publique les abus pratiqués sur le parquet (trading floor). En 1909, le Hughes Committee de l'État de New York enquête sur les pratiques spéculatives. En 1912, le Pujo Committee mandaté par le Congrès fait de même et critique la spéculation largement répandue au NYSE. En 1916, Louis D. Brandeis publie son célèbre brûlot dirigé contre l'establishment bancaire: Other People's Money and How the Bankers Use It. À l'époque, les membres du NYSE ne sont pas accoutumés à voir leurs affaires internes ouvertement révélées. Ils craignent surtout que le pouvoir fédéral n'entreprenne de contrôler leurs activités. En effet, la Bourse de New York est encore une association privée auto-réglementée (self-regulated) et, au fur et à mesure que s'affirme son rôle de marché financier central, une contradiction gênante grandit entre son statut privé et sa fonction publique. Des députés, des journalistes et des leaders d'opinion se demandent si le NYSE sert prioritairement les intérêts de ses membres ou les intérêts du grand public. Bon nombre d'entre eux proposent que le marché soit directement réglementé par le gouvernement fédéral pour s'assurer que les intérêts de tous les investisseurs américains soient correctement protégés. C'est ce que les membres du NYSE veulent éviter à tout prix et les efforts de relations publiques qu'ils déploient dans les années 20 s'inscrivent dans une stratégie de défense de leur autonomie.

Pour repousser la menace d'un contrôle exercé par Washington, les officiels du NYSE prennent de nombreuses initiatives pour mettre l'opinion publique de leur côté. Ils créent un Committee on Publicity (COP), organisent de multiples manifestations promotionnelles et mettent sur pied des organes de relations publiques. Ainsi, le Better Business Bureau (BBB) a pour mission d'améliorer la déontologie des professionnels de la Bourse. Des programmes de publications éducatives et de visites commentées du parquet, des conférences et des projections de films aux 
quatre coins des États-Unis doivent redorer le blason du NYSE (Ott 2004 : p. 25). L'initiative la plus remarquable est cependant la campagne promotionnelle présentant la Bourse de New York comme un marché libre et ouvert. Elle est portée par le slogan «The NYSE is the Free and Open People's Market» adopté en 1925. Les objectifs déclarés de la campagne sont de donner une image positive du NYSE, d'éduquer les citoyens pour qu'ils investissent avec profit dans les actions des grandes sociétés cotées. Il s'agit également de contribuer à l'émergence d'une nation d'actionnaires (a nation of shareholders) et d'harmoniser les idéaux de la démocratie et les bienfaits de l'économie libérale.

Au début de la décennie, le NYSE a engagé un économiste, J. Edward Meeker, pour qu'il rédige un ouvrage de fond expliquant et justifiant le fonctionnement de la Bourse de New York. En 1922, il achève The Work of the Stock Exchange, un livre de plus de 700 pages qui représente l'effort le plus abouti jusque-là pour légitimer le rôle économique du NYSE. En particulier, Meeker explique de façon détaillée le sens de l'expression «free and open market», proposée en 1914 par l'économiste Henry C. Emery (Meeker 1930 : p. 687; Ott 2004 : p. 13). Selon lui, «free» signifie que la Bourse ne peut être dominée par un seul opérateur ou par un groupe d'opérateurs et «open» veut dire que toute transaction, modeste ou importante, est effectuée ouvertement et donne lieu à un prix connu de tous, le cours de l'action, où s'équilibrent l'offre et la demande (Meeker 1930 : p. 48-49). L'économiste du NYSE fonde ainsi le sens officiel explicite du slogan de la campagne, sens qui pourrait être résumé comme suit: le NYSE est un marché qui ne peut être manipulé par une minorité et dont le fonctionnement est transparent. En revanche, Meeker ne définit nulle part l'expression people's market mais nous observons pour le moment que l'ajout de «people's» au slogan ne paraît guère poser de difficulté d'interprétation. Le sens semble être: le NYSE est au service du peuple américain. Le contexte historique étant décrit et le contenu explicite du slogan posé, peut-on dire que l'énoncé comporte également un contenu implicite?

\section{Le décodage du contenu implicite du message: points de méthode}

Catherine Kerbrat-Orechionni pose la question préalable à toute enquête sur l'au-delà de l'explicite d'un énoncé: où commence le domaine de l'implicite? (op. cit. p. 21). Avant de répondre, elle pose comme postulat que «toute unité de contenu susceptible d'être décodée possède nécessairement dans l'énoncé un support linguistique quelconque» (op.cit. p. 13). Si ce contenu implicite supposé existe bien, il doit donc avoir un support linguistique dans l'énoncé «The NYSE is the Free and Open People's Market». Dans ce cas, ce support ne peut se trouver que dans les mots the, free, open et people's qui qualifient market. 
Par ailleurs, C. Kerbrat-Orechionni précise qu'interpréter un énoncé consiste pour l'allocutaire (celui qui le reçoit) à appliquer ses diverses « compétences » aux signifiants inscrits dans la séquence de manière à en extraire des signifiés (op. cit. p. 162). Parmi ces compétences, elle distingue les compétences linguistique, encyclopédique, logique et rhétorico-pragmatique. Le message que nous examinons ne se situe ni dans un contexte d'échange dialogué, ni dans une situation concrète de la vie quotidienne, ni enfin dans le cadre d'une démonstration argumentée. Il est émis dans l'environnement socio-économique général des États-Unis au cours des années 20. Ce sont donc prioritairement les compétences dites encyclopédiques qu'il nous faut mobiliser pour l'interpréter.

\section{Le décodage des présupposés implicites}

Dans sa recherche sur la nature de l'implicite, C. Kerbrat-Orechionni distingue les présupposés des sous-entendus. Un présupposé «est une unité de contenu qui doit être nécessairement vraie pour que l'énoncé qui la contient puisse se voir attribuer une valeur de vérité» (op. cit. p. 27). Or le slogan «The NYSE is the Free and Open People's Market» a la forme d'une assertion exprimant une vérité générale qui ne souffre aucune contestation. La proposition inclut donc au minimum un présupposé implicite qui pourrait prendre les formes suivantes:

[Everyone knows that] The NYSE is the Free and Open People's Market. Every American is aware that...

All Americans agree that...

We all accept that...

It is widely acknowledged that...

It is true that...

À l'époque où le slogan est proposé au public américain, ce présupposé est loin d'être universellement reconnu comme vrai. Les compétences encyclopédiques que nous fournit l'histoire de la période nous indiquent clairement que les multiples critiques émises à l'encontre du NYSE, critiques qui l'ont précisément encouragé à lancer sa campagne, contestent toutes les propositions du slogan. En effet, il peut se voir opposer quatre démentis qui remettent en question chacun des qualifiants qui sont attribués à la Bourse, à l'exception probable de market puisque tout le monde peut aisément convenir qu'une bourse est bien un marché. Nous citons en support certains éléments encyclopédiques qui fondent notre compétence interprétative. 
Contestation $\mathrm{n}^{\circ} 1$ des présupposés: remise en question de the: The NYSE is not the only free and open people's market.

L'utilisation de l'article the indique une application exclusive des attributs free, open et people's au NYSE, et cette exclusivité est contestable. À l'époque, vingt-neuf bourses concurrentes opèrent aux États-Unis et la plupart d'entre elles peuvent se targuer d'être aussi ouvertes et libres que le NYSE (Galbraith 1961 : p. 83; Kamm 1942 : p. 97). Au minimum, la proposition exige d'être prouvée avant d'accéder au statut de vérité incontestable.

Contestation $\mathrm{n}^{\circ} 2$ des présupposés: remise en question de free: The NYSE is not a free market.

Les manipulations du marché par les cliques de haussiers (bulls) et de baissiers (bears) font incontestablement partie de l'histoire du NYSE ainsi que l'indique son ouvrage officiel, The New York Stock Exchange, rédigé par l'un de ses membres (Stedman 1905 : p. 155-260). Il est tout aussi inexact de dire que ce marché ne peut être dominé par des groupes d'intérêt. Le tout récent scandale du Stutz Corner en 1920 où le spéculateur Allan A. Ryan avait manipulé le cours de l'action Stutz attestait, peu de temps avant la campagne, que le marché pouvait être manipulé par un seul individu (Ott 2004 : p. 16-22). Le NYSE ne peut donc, sans démonstration solide, satisfaire à la définition donnée par son économiste J.Edward Meeker reproduite en début d'article.

Contestation $\mathrm{n}^{\circ} 3$ des présupposés: remise en question de open: The NYSE is not an open market.

La distinction entre les initiés de la bourse (insiders) et les non initiés (outsiders) est largement acceptée depuis les premiers écrits sur le NYSE en 1870 (Medbury 1968 : p. 152-206) et elle relativise l'ouverture de ce marché. Sa transparence est également remise en question par de nombreux témoignages sur l'ambiance de secret et de mystère qui y règnait. L'économiste Meeker y fait lui-même allusion (Meeker 1930 : p. 477478 ) et plusieurs ouvrages publiés après la campagne indiquent que l'atmosphère confidentielle qui imprégnait la Bourse n'était pas en voie de disparition à l'époque mais qu'elle a au contraire perduré pendant toute la décennie (Sparling 1930; Parker 1932). 
Contestation $\mathrm{n}^{\circ} 4$ des présupposés: remise en question de people's: The NYSE is not the people's market.

Au début des années 20, seule une petite minorité d'Américains aisés intervenait régulièrement en bourse même si Wall Street commençait à alimenter les conversations quotidiennes. En 1934, une enquête du Sénat estime à $5 \%$ environ le nombre d'actionnaires actifs avant le krach de 1929. Au début de la décennie, cette proportion était certainement bien inférieure et plusieurs études relativisent la dimension populaire de l'activité boursière dans les années 20 (Ott 2004 : p. 5; Galbraith 1961 : p. 83).

La forme assertive du slogan suggère donc que les propositions dont il est porteur faisaient l'objet d'un large consensus à l'époque où il a été émis alors que de nombreux documents montrent que ce consensus était très relatif dans le meilleur des cas. Notre hypothèse que le slogan comporte un contenu implicite se trouve donc confirmée. Son message «amène l'allocutaire à penser quelque chose». En l'espèce, il l'encourage à donner son aval à la proposition globale de l'énoncé qui est présentée comme incontestable. Notre hypothèse est également confirmée dans le cas contraire, c'est-à-dire si l'allocutaire conteste ces présupposés. En effet, le message comporte alors un autre présupposé implicite qui pourrait être formule ainsi: «Contrary to what you think, the NYSE is...», ou encore «You believed the NYSE was not free, not open and not the people's market, but it is». Dans le contexte difficile des années 20 où le NYSE doit sortir de sa réserve pour défendre sa réputation, le slogan apparaît donc soit comme une affirmation implicitement posée comme consensuelle, soit comme une réponse à des contestations éventuelles. De multiples sens implicites présupposés débordent donc son contenu explicite.

\section{Le décodage des sous-entendus implicites}

Après avoir étudié les présupposés implicites du message, nous examinons s'il présente des sous-entendus implicites. C. Kerbrat-Orechionni les définit comme suit: la classe des sous-entendus «englobe toutes les informations qui sont susceptibles d'être véhiculées par un énoncé donné, mais dont l'actualisation reste tributaire de certaines particularités du contexte énonciatif» (op. cit. p. 39). D'après elle, l'interprétation des sous-entendus exige de la part de l'allocutaire «un calcul interprétatif» intimement lié aux circonstances de l'énonciation ainsi que les quatre compétences déjà indiquées plus haut. Un examen intuitif du 
slogan nous laisse percevoir, au-delà de son sens explicite, certaines de ces informations sous-entendues. Par exemple, la présentation du NYSE comme «the Free and Open People's Market» semble intuitivement valorisante dans le contexte américain. Une connaissance élémentaire de la civilisation américaine confirme ces intuitions et révèle des connotations positives dans les qualifiants free, open et people's. Manifestement, les artisans du slogan ont souhaité présenter leur association sous le meilleur jour possible en montrant qu'elle travaille en harmonie avec les grandes valeurs fondatrices du pays. En particulier, les mots free et people sont chargés d'histoire glorieuse et lourds de sens positif dans le contexte américain. Ils figurent dans de nombreux slogans, chants et textes fondateurs qui ont contribué à la naissance de la nation. People et liberty sont notamment inscrits dans le préambule de la constitution des États-Unis. Open semble moins consacré par l'histoire que free et people mais de nombreuses références encyclopédiques peuvent facilement confirmer qu'open est généralement connoté positivement dans le contexte américain. Un des exemples qui étayent cette acception est le discours qu'Otto Kahn prononça en 1917 devant ses collègues du NYSE pour les encourager à communiquer avec l'opinion publique. Les extraits suivants montrent clairement combien l'ouverture (exposure to the open air) est positivement connotée dans la société démocratique américaine tandis que le repli sur soi (seclusion) est négativement connoté.

This is the age of publicity, whether we like it or not. Democracy is inquisitive and won't take things for granted [...] Business and businessmen must come out of their old time seclusion... [...] No bealthy organism is hurt by exposure to the open air. [...] Democracy wants to be shown. [...] [The Stock Exchange] should not only not avoid the fullest publicity and scrutiny, but it should welcome and seek them. It has nothing to bide and it should be glad to show that it has notbing to bide. (Kahn 1917: p. 26-27)

Si les connotations positives des trois qualifiants semblent avérées, nous remarquons néanmoins une certaine hétérogénéité dans leurs différents contenus sémantiques. Free et open ont un sens technique et économique précisément défini par J. Edward Meeker (op. cit. p. 687) tandis que, comme nous l'avons indiqué, people's market n'a pas de définition économique. Sa présence à la suite de free et d'open apparaît donc comme rapportée et artificielle. Elle ne semble se justifier que par la connotation positive qu'elle apporte au slogan. Autrement dit, les qualifiants free et open ont un contenu explicite (technique) et un contenu implicite (connotatif) tandis que people's n'a qu'un contenu implicite (connotatif). Cette distinction met en évidence le glissement sémantique du domaine économique au domaine politique que le slogan effectue en s'ouvrant sur deux termes techniques et en se concluant sur un terme non technique 
mais puissamment symbolique. En effet, dans le champ sémantique de la politique américaine, le mot people a un pouvoir signifiant inégalé. Dans le préambule de la constitution, la formule sacrée «We the People of the United States...» reconnaît au peuple la légitimité politique ultime. Du coup, par une sorte de contamination remontante, le sens profondément politique de people's reflue sur free et open dont le sens technique s'efface au profit des connotations politiques positives qu'ils peuvent porter.

Ce décodage révèle que le fond du message ne porte pas sur les caractéristiques économiques du marché mais sur ses caractéristiques politiques. Présenter le NYSE comme «le marché du peuple» donne à sa légitimité une fondation incontestable. Cette légitimité est certes justifiée dans le domaine économique par les qualifiants free et open qui disposent d'une définition technique apportée par Meeker. Mais nous comprenons grâce à people's que cette légitimité a surtout un caractère politique puisqu'elle est présentée comme démocratique. Dans cette perspective, nous percevons le rôle de l'implicite dans le slogan: il consiste à amalgamer l'économique et le politique afin de poser le NYSE comme le centre d'une démocratie économique tout aussi légitime que le système politique lui-même. Meeker confirme définitivement notre interprétation en écrivant par ailleurs: «A stock exchange is the most democratic type of market» (Meeker 1930 : p. 49; Ott 2004 : p. 5, 30).

\section{Finaliser le décodage des sous-entendus à l'aide de la compétence logique}

Parmi les quatre compétences nécessaires à l'interprétation des contenus implicites, la compétence logique permet à l'allocutaire de reconstituer les raisonnements sous-jacents à un énoncé et particulièrement d'en comprendre la «logique naturelle» (Kerbrat-Orechionni 1986 : 165194). Les raisonnements implicites reposent généralement sur des structures syllogistiques enfouies qui permettent à l'allocutaire d'inférer certaines conclusions à partir de postulats majeurs et mineurs du type:

(i) Majeure: Un tel ne vient me voir que lorsqu'il a des ennuis (donc par intérêt).

(ii) Mineure: Or un tel est venu me voir.

(iii) Conclusion: Donc il a des ennuis. (op. cit. p. 165).

Dans le cas de notre slogan, nous pouvons inférer de l'analyse précédente le raisonnement sous-jacent représenté au tableau cidessous.

Logique implicite du slogan

PHASES DE LA DÉMONSTRATION

Majeure

\section{CONTENUS IMPLICITES}

Le système démocratique est le meilleur. 
Mineure (le slogan lui-même)

Conclusion
Or, le NYSE est le marché du peuple Donc, le NYSE est le meilleur type de marché possible.

Dans ce raisonnement, le slogan ne constitue que la mineure et il revient à la compétence logique de l'allocutaire de reconstituer la majeure et d'inférer la conclusion qui constitue par conséquent le message promotionnel profond du slogan. Dans ce cas-là encore, notre compétence encyclopédique confirme que la conclusion implicite à laquelle aboutit le raisonnement enfoui du slogan est bien l'excellence du NYSE en tant que marché démocratique. Lorsqu'il fut interrogé entre 1933 et 1934 par les enquêteurs du Sénat sur la nature de la Bourse de New York, son président Richard E. Whitney, déclara que c'était une institution parfaite («a perfect institution») (Pecora 1939 : p. 259).

Néanmoins, nous estimons que la conclusion à laquelle nous aboutissons n'épuise pas totalement les sous-entendus implicites du slogan. C. Kerbrat-Orechionni signale que les compétences logiques permettent de réaliser certaines inférences qu'elle appelle «inférences praxéologiques». Ces inférences apportent des «informations présupposées ou sous-entendues par l'énoncé de tel ou tel fait diégétique, qui au nom d'une "certaine logique des actions" [...] impliquent nécessairement ou éventuellement la réalisation d'autres actions nécessairement ou éventuellement corrélées» (op. cit. p. 189-190). Nous émettons l'hypothèse qu'il existe un raisonnement sous-jacent supplémentaire sous le raisonnement implicite déjà exposé et que ce raisonnement repose sur une logique d'action de la vie quotidienne exprimée par des dictons tels que:

Leave well alone. (Le mieux est l'ennemi du bien.)

If it ain't broke don't fix it.

Don't change a winning team.

Ces scénarios d'action disent en bref qu'il faut éviter de modifier ce qui fonctionne déjà correctement au risque d'aboutir à un résultat inférieur. À partir de cette logique d'action, le raisonnement praxéologique suivant peut être inféré du slogan comme le montre le tableau cidessous.

\section{Démonstration praxéologique implicite}

PHASES DE LA DÉMONSTRATION

Majeure

Mineure

Conclusion

\section{CONTENUS IMPLICITES}

Ce qui est parfait ne peut être modifié sans perdre de sa perfection

Or, le NYSE est parfait.

Donc, il ne faut pas le modifier 
Nous estimons que les deux raisonnements ne sont pas en concurrence dans le message mais que, au contraire, ils se complètent et s'emboîtent logiquement l'un dans l'autre. La double conclusion finale est donc non seulement que le NYSE est le meilleur marché possible mais qu'il ne faut pas essayer d'interférer avec son fonctionnement ou d'altérer sa nature. Le tenter pourrait menacer la démocratie du marché que les Américains méritent. Il est clair que ce message s'adresse moins au grand public américain qu'aux politiciens désireux de réglementer la Bourse de New York. Il s'inscrit dans l'intense campagne de lobbying entreprise par le NYSE depuis la fin du XIXe siècle pour éviter que la législature de l'État de New York et que le Congrès fédéral ne supervisent ses activités jusqu'alors strictement privées. Le contenu implicite du message cherche un soutien auprès de l'opinion publique en présentant le NYSE comme libre, ouvert et au service du peuple. Mais ce faisant, il asservit ce soutien populaire à ses intérêts et l'utilise comme caution afin de contrecarrer les efforts régulateurs du pouvoir fédéral.

\section{Pourquoi avoir recours à l'implicite?}

Catherine Kerbrat-Orechionni s'interroge sur la fonction des contenus implicites. Elle répond que, malgré les contraintes linguistiques et interprétatives qu'impose son élaboration, l'implicite est généralement utilisé pour des raisons de convenance, afin de déjouer certaines censures d'ordre moral, politique ou juridique, et pour contourner certains tabous (op. cit. p. 277). Dans la campagne promotionnelle étudiée ici, il n'est pas difficile d'identifier les censures et les tabous possibles qui imposent un recours à l'implicite. Les officiels du NYSE peuvent certes présenter leur institution comme une démocratie économique mais ils ne peuvent contester ouvertement le droit de regard des autorités fédérales démocratiquement élues par le peuple. Le prestige du système politique américain est si grand qu'il est tabou de s'y opposer de front par des énoncés explicites. En usant des formes diverses du présupposé, du sous-entendu et de la suggestion, le NYSE use donc largement de l'implicite pour mettre l'opinion publique de son côté face aux législateurs de Washington sans pour autant remettre en question leur légitimité. Le slogan dit, tout en se réservant la possibilité de prétendre qu'il n'a pas dit. Un texte écrit par E. H. H. Simmons, le président du NYSE dans les années 20, illustre bien la lutte intense mais indirecte de la Bourse contre la mainmise des politiques. Les autorités fédérales n'y sont jamais explicitement mentionnées mais elles sont constamment évoquées et opposées au peuple américain et à sa sagesse. Les procédures de création de l'implicite y sont similaires à celles de notre slogan. 
Economic soothsayers or any small group of experts [only seek] autocratic control over our business lives. The American people are neither mentally nor morally unfit to manage the prosperity they have created. I refuse to believe [they] are less able to exercise the prerogatives of economic and financial freedom than they are to use wisely the political freedom that was won a century and a half ago... We do not need panaceas or artificial legislation. Mere pride of supposedly expert opinion must yield place before the bard facts of a new society. The universal thrift and intelligence which bave created the progress and prosperity for American business will continue to manage and administer it. (E. H. H. Simmons, président du NYSE (1929), cité par Ott 2004 : p. 32).

Notons que par deux fois Simmons utilise la première personne du pluriel (our et we) sous-entendant que le NYSE et le peuple américain parlent de la même voix et que ni l'un ni l'autre ne veulent entendre parler de législation artificielle. Notons également que, comme dans le slogan, Simmons amalgame liberté économique (economic and financial freedom) et liberté politique (political freedom) afin de suggérer que limiter la première (autocratic control over our business lives) équivaudrait à limiter la seconde.

Vers la fin de la décennie, un court métrage promotionnel produit par le NYSE, The Nation's Marketplace (1928), eut recours à des contenus implicites similaires pour faire passer le même message que le slogan «The NYSE is the Free and Open People's Market». Les premières images du film «nationalisent» et sacralisent Wall Street en présentant les plus imposants monuments de la rue: la colonnade classique du bâtiment du U.S. Subtreasury ornée de la statue de George Washington, l'église de Trinity Church, les chapitaux corinthiens de la Bourse. La suite évoque ce qu'ont réalisé les marchés financiers (What the capital markets wrought) grâce à des images de hauts-fourneaux et d'usines gigantesques en pleine activité. Enfin, le déroulement d'une transaction boursière typique est décrit: un certain M. Blair de Tacoma dans l'État de Washington téléphone à son courtier pour qu'il lui achète 200 actions de la société High Mountain tandis qu'un résident de la Nouvelle-Orléans donne un ordre de vente symétrique. La machinerie efficace du NYSE effectue promptement l'échange et publie un cours mis à la disposition du marché. Une carte des États-Unis montre que les deux ordres, émis à des milliers de kilomètres l'un de l'autre, se rencontrent et se compensent mutuellement à New York, «the Nation's Marketplace», avec une facilité déconcertante. Le message implicite du film est que New York est la capitale économique du pays et que, dans le parfait mécanisme qu'elle orchestre, Washington, qui ne figure pas sur la carte et qui n'y joue aucun rôle, n'a surtout aucun rôle à y jouer. 


\section{L'implicite dans la campagne «Own Your Share of American Business» (1954-1970)}

\section{Le contexte de la campagne}

À la suite du krach de 1929 et de la Grande Dépression des années 1930, le NYSE subit l'hostilité de l'opinion publique et la détermination des autorités fédérales à réglementer ses activités. La création de la Securities and Exchange Commission (SEC) en 1934 marque la défaite de la Bourse de New York dans la défense de son indépendance par rapport à Washington. Non seulement ses officiels durent-ils répondre de leurs actes lors des humiliantes sessions des Pecora Hearings (1933-34), mais leur inspirateur, l'ancien président du NYSE Richard Whitney, fut lui-même emprisonné au pénitencier de Sing-Sing pour malversations en 1938 (Geisst 2000 : p. 57). Lors de l'embellie économique consécutive à la fin du second conflit mondial, le NYSE engage en 1951 un nouveau président, G. Keith Funston, pour redynamiser son activité alors encore inférieure à son niveau de 1929. Sous sa direction, la Bourse lance une campagne promotionnelle identifiée par le slogan «Own Your Share of American Business ». L'objectif n'est plus de rivaliser avec le Congrès comme dans les années 20 mais de redonner confiance aux Américains pour qu'ils reviennent vers Wall Street. La campagne doit débarrasser la Bourse de son image de casino et présenter l'investissement comme un moyen légitime et moral de s'enrichir (Traflet 2003 : p. 3). En outre, en pleine guerre froide, encourager les employés et les ouvriers à devenir des capitalistes prospères constitue une manière bien américaine de mener la bataille idéologique contre le bloc de l'Est (Traflet 2003 : p. 5). Mis au point en 1953 par Ruddick C. Lawrence, un expert en publicité qui avait travaillé pour le magazine Fortune, le slogan apparut l'année suivante dans des journaux, sur des affiches, des tracts, des publications d'entreprise, des placards promotionnels en magasins. Il devint l'un des messages de communication de masse les plus connus de son époque (Traflet 2003 : p. 7).

\section{L'analyse des présupposés implicites}

Own + impératif: une forme d'«empowerment» grammatical

«Own Your Share of American Business» se distingue du slogan précédemment étudié en ce qu'il établit des relations différentes entre le locuteur et l'allocutaire. Dans l'assertion «The NYSE is the Free and Open People's Market », une vérité absolue et incontestable était énoncée in abstracto et elle n'établissait aucune relation particulière entre l'émetteur et le destinataire. En revanche, «Own Your Share... » s'adresse personnellement à l'allocutaire et lui donne un ordre exprimé par l'impératif. Bien 
que simple, cet ordre n'est pas très clair et il pose un certain nombre de problèmes de compréhension. D'après C. Kerbrat-Orechionni, l'interprétation de tout énoncé repose d'abord sur la compétence linguistique des sujets parlants: «Il n'est donc aucune unité de contenu dont le décodage puisse s'effectuer sans l'intervention de la compétence linguistique $\gg$ (op. cit. p. 162). Dans le cas présent, la compétence linguistique des allocutaires anglophones auxquels le message est destiné leur indique que to own est un verbe qui décrit l'état d'être possesseur de quelque chose. L'Oxford English Dictionary donne comme équivalent: «to have or to bold as one's own, possess». Les grammairiens Thomson et Martinet indiquent que own appartient à un groupe de verbes exprimant la possession, tels que belong, owe, possess, qui ne prennent généralement pas la forme be + ing (1981 : p. 142). Ils distinguent d'ailleurs entre les verbes qui expriment des actions délibérées et ceux qui expriment des actions involontaires (deliberate/involuntary verbs). Ils observent que les verbes exprimant des actions involontaires ne prennent généralement pas la forme be + ing (id. p. 142) Cette explication peut a fortiori s'appliquer à des verbes exprimant un état qui ne résulte pas d'une action volontaire du sujet. Henri Adamczewski approfondit l'analyse en expliquant que les «verbes d'état» comme know, belong, cost, contain, resemble, understand, want,... ne sont pas des verbes à «sujet agent» comme write, walk, drink, read. D'après lui, ils ne prennent pas la forme be + ing parce qu'ils ne «présentent pas de manifestation repérable en situation» (Adamczewski \& Gabilan 1992 : p. 36).

Ces observations sur la forme verbale be + ing peuvent être étendues à l'usage de l'impératif pour cette catégorie de verbes. En effet, pour les raisons énoncées ci-dessus, il est difficile de concevoir qu'un énoncé émette un ordre adressé à un sujet à propos d'un état qui échappe à sa capacité d'action. La compétence linguistique des anglophones les dissuade d'utiliser l'impératif pour cette catégorie de verbes. Les énoncés exprimant un ordre comme Possess!, Owe!, Belong!, Cost!, Resemble!, Want!, paraissent rares et dénués de sens. La même remarque peut s'appliquer à l'impératif Own! du slogan étudié. Le sujet de cet impératif ne peut volontairement décider d'être le propriétaire d'un objet, que cet objet lui appartienne effectivement ou non. L'état de propriétaire ne pouvant faire l'objet d'une «manifestation repérable en situation » selon la formule d'Adamczewski, le verbe own se prête mal à la réalisation d'énoncés porteurs de formes en be + ing et de formes impératives tels que I can see he is owning this house et Own this house!

En mettant own à la forme impérative, le slogan use donc d'un présupposé linguistique qui heurte la compétence ordinaire de l'allocutaire anglophone. En prenant des libertés avec les contraintes habituelles de la langue, il présuppose que own n'est pas un verbe d'état exprimant une 
situation subie mais un verbe d'action reposant sur la volonté d'un sujet agent. Autrement dit, en mettant le verbe à un mode de conjugaison inusité, le slogan redonne au sujet un peu de sa capacité décisionnelle. Grâce à une forme subtile d'«empowerment» grammatical, il lui suggère qu'il dispose d'un pouvoir imprévu, pouvoir qu'il l'encourage à exercer.

\section{«Your Share of American Business» : jeu de mot et patriotisme économique}

Dans une large mesure, le contenu implicite du slogan repose sur le jeu de mot suscité par l'usage de your share. Bien l'interpréter repose sur une compétence hybride, à la fois encyclopédique et linguistique. En langage courant, your share (ta/votre part) désigne, d'après les dictionnaires, the part belonging to you, owed to you (la part qui te/vous revient) et ce contenu conduit à l'interprétation la plus large et la plus ordinaire du message. Néanmoins, les allocutaires rompus à l'anglais spécialisé de la bourse disposent des compétences encyclopédiques et linguistiques nécessaires pour interpréter également share comme a part of the capital of a publicly quoted company, a share of stock (une part du capital d'une société cotée, une action). Dans l'énoncé, your share signifie donc à la fois «la part qui vous revient» et «votre action boursière». Le jeu de mot fait fusionner les deux sens et l'allocutaire comprend simultanément «qu'une part est à lui » et que «l'action boursière est à lui ».

En outre,... of American Business spécifie de quelle part il s'agit dans le message: une part de l'activité économique américaine. Néanmoins, le syntagme American Business recouvre une réalité si diffuse qu'il est difficile de bien comprendre ce qu'il désigne et de quelle part il s'agit. Pour l'allocutaire individuel et d'après ce que nous avons proposé plus haut, Your Share of American Business peut avoir les deux sens suivants: (i) ce qui lui revient dans les affaires où il intervient (sens général); (ii) les actions de sociétés qu'il détient (sens boursier). Toutefois, l'étendue sémantique d'American business peut mener l'allocutaire à une interprétation beaucoup plus large, telle que: «vos activités économiques individuelles et/ou vos actions font partie de l'activité économique américaine dans son ensemble et contribuent à l'enrichissement du pays; elles représentent la part de l'Amérique qui vous revient en tant qu'Américain ». Dans le slogan, le syntagme American business opère donc comme une double synecdoque: la partie (les activités individuelles et/ou les actions du sujet) est mise pour le tout (l'activité économique américaine dans son ensemble); ou inversement, le tout est mis pour la partie. Dans les deux cas, le contenu implicite est que l'allocutaire se voit alloué un rôle à dimension nationale (ce que vous faites contribue à l'activité économique du pays et inversement, son activité économique dépend de chacun de ses citoyens, donc de vous). 
Tout Américain correctement intégré dans son environnement socio-culturel peut être sensible au caractère patriotique de ce message. Une compétence encyclopédique élémentaire suffit pour replacer l'énoncé dans le vaste ensemble des discours qui présentent l'Amérique comme la nation par excellence du travail et des affaires. Ces discours sont aussi anciens que l'Amérique elle-même et ils ont généré une culture populaire de dictons, de citations et de proverbes qui contribuent à façonner la civilisation américaine. Citons par exemple:

America is the Land of Labour. (Benjamin Franklin, 1782)

Time is money. (Benjamin Franklin in Cohen \& Cohen 1992 : p. 168)

To Americans, business is everything. [...] Business is the very soul of an American. [...]

No admission bere [to America] except on business. (Grund 1996: p. 190-191)

The chief business of the American people is business. (Calvin Coolidge 1925 in Cohen \& Cohen 1992 : p. 122)

Everybody ought to be rich. (John J. Raskob, 1929 in Geisst 2000 : p. 22)

America is a nation of shareholders. (dicton répandu à partir des années 20) (Ott 2004 : p. 5, 30)

Le slogan «Own Your Share of American Business» semble prolonger cet ensemble discursif et émaner naturellement de la culture entreprenariale américaine dans laquelle All Americans are businessmen. Il suggère que tout Américain participe à l'activité économique de son pays et qu'à ce titre, il a droit, en tant qu'Américain, à sa part légitime d'American business.

\section{Deux présupposés contradictoires}

Catherine Kerbrat-Orechionni montre que les énoncés en forme d'ordre ou de requête visent à opérer des transformations de situations dans la vie réelle et qu'ils impliquent généralement des présupposés à la fois logiques et pragmatiques. Ainsi, l'ordre Open the door suppose, pour que l'énoncé ait un sens et pour qu'il soit effectivement suivi d'un résultat, que la porte dont il est question soit fermée au moment où il est émis (op.cit. p. 36). Suivant cette logique, l'ordre Own your share... implique le présupposé logico-pragmatique You don't own the share... puisqu'il se propose de le modifier. Mais par ailleurs, la partie d'énoncé your share implique le présupposé inverse qui pourrait prendre les formes: you own the share, the share belongs to you, the share is yours. L'analyse fait donc apparaître que l'énoncé «Own Your Share of American Business» repose sur deux présupposés contradictoires exprimés par own d'un côté et your de l'autre:

(i) Présupposé négatif: You don't own the share.

(ii) Présupposé positif: You own the share. 
En conséquence, le slogan semble enjoindre à l'allocutaire de posséder ce qui lui appartient déjà et il suscite en lui l'impression étrange que deux messages incompatibles lui sont envoyés simultanément. Ce sentiment s'apparente à un phénomène que les psychologues nomment « dissonance cognitive $\gg$. Un sujet se trouve en situation de dissonance cognitive lorsqu'il est mis en contradiction avec lui-même (Shiller 2001 : p. 1314). Il en éprouve un certain malaise qu'il tente de dissiper en adoptant des comportements à même d'évacuer la dissonance. Dans le cas présent, le malaise vient du fait que le message reconnaît au sujet un statut légitime de propriétaire (Because you are an American, you own a share of American business) et que dans le même temps il implique que ce statut n'existe pas puisqu'il ordonne au sujet d'être propriétaire. Le slogan perturbe le sujet en l'enfermant dans une contradiction désagréable. Comme dans les cas de dissonance cognitive, il tente d'échapper à la contradiction en cherchant une porte de sortie. Le slogan lui suggère implicitement une solution mais celle-ci n'apparaît clairement qu'au terme du décodage de sa logique implicite sous-jacente.

\section{Décodage de la logique implicite du slogan}

La logique implicite du slogan se décompose en trois démonstrations distinctes. Dans les trois cas, la proposition majeure implicite du slogan est All Americans are businessmen mais les suites varient en fonction des sens donnés à share et du présupposé positif ou négatif adopté. Elles aboutissent successivement à des solutions non satisfaisantes pour le sujet jusqu'à ce qu'il parvienne à la solution (3) seule capable de dissiper son malaise (Tableaux 2.1., 2.2., 2.3.).

Tableau 2.1. - Logique implicite du slogan: présupposé positif; sens indéterminé de share

$\begin{array}{lll}\begin{array}{l}\text { PHASES DE LA } \\ \text { DÉMONSTRATION } \\ \text { Majeure }\end{array} & \text { CONTENUS IMPLICITES } & \text { SENTIMENTS DU SUfET } \\ \begin{array}{l}\text { Mineure (1): } \\ \text { présupposé positif }\end{array} & \begin{array}{l}\text { You own a share } \\ \text { of American business }\end{array} & \text { Vérité incontestable } \\ \text { Conclusion (1) } & \text { So, you are a real American } & \begin{array}{l}\text { Sentiment de satisfaction et de } \\ \text { normalité }\end{array} \\ \text { Solution (1) } & \begin{array}{l}\text { Own a share of American } \\ \text { business because it is yours }\end{array} & \begin{array}{l}\text { Solution contradictoire } \\ \text { en elle-même et contraire à la } \\ \text { conclusion (1): dissonance } \\ \text { cognitive }\end{array}\end{array}$

Dans le Tableau 2.1., la mineure a un présupposé positif et le sens de share n'est pas précisé. La conclusion (1) donne au sujet un sentiment de 
satisfaction et de normalité. Cependant, la solution (1) donnée par le slogan est contradictoire en elle-même et contraire à la conclusion (1): elle laisse l'allocutaire en situation de dissonance cognitive. Il a le sentiment d'être un véritable Américain et le slogan lui donne un ordre suggérant qu'il n'en est rien. Cette démonstration correspond en gros à la première perception du message par un allocutaire qui le découvre.

Tableau 2.2. - Logique implicite du slogan: présupposé négatif; sens général de share (une part)

\begin{tabular}{|c|c|c|}
\hline $\begin{array}{l}\text { PHASES DE LA } \\
\text { DÉMONSTRATION }\end{array}$ & CONTENUS IMPLICITES & SENTIMENTS DU SUfET \\
\hline Majeure & All Americans are businessmen & Vérité incontestable \\
\hline $\begin{array}{l}\text { Mineure (2): } \\
\text { présupposé négatif }\end{array}$ & $\begin{array}{l}\text { You own no share } \\
\text { of American business }\end{array}$ & \\
\hline Conclusion (2) & So, you are not a real American & $\begin{array}{l}\text { Sentiment d'anormalité, } \\
\text { d'injustice et d'aliénation: } \\
\text { dissonance cognitive }\end{array}$ \\
\hline Solution (2) & $\begin{array}{l}\text { Be a real American } \\
\text { by owning a share of } \\
\text { American business }\end{array}$ & $\begin{array}{l}\text { Sortie possible de la dissonance } \\
\text { cognitive mais, à cause du sens } \\
\text { général de share, l'ordre reste } \\
\text { vague }\end{array}$ \\
\hline
\end{tabular}

Dans le Tableau 2.2., la mineure a un présupposé négatif et share a son sens général (une part). Dans ce cas, la conclusion (2) est qu'un Américain qui ne possède aucune part dans l'activité économique américaine n'est pas un vrai Américain. Cette interprétation crée un sentiment d'anormalité, d'injustice et d'aliénation car le sujet se sent dépouillé à tort de son identité américaine légitime. Le slogan lui propose la solution (2) pour restaurer la normalité, recouvrer son identité américaine et rentrer dans son droit. Néanmoins, le sens général de share est trop vague pour donner à l'ordre un sens clair et précis. Au cours de cette démonstration, le sujet commence à interpréter le message mais il n'aboutit à aucune solution lui permettant de dissiper son malaise.

Tableau 2.3. - Logique implicite du slogan: présupposé négatif; sens boursier de share (une action)

$\begin{array}{lll}\begin{array}{l}\text { PHASES DE LA } \\ \text { DÉMONSTRATION }\end{array} & \text { CONTENUS IMPLICITES } & \text { SENTIMENTS DU SUFET } \\ \begin{array}{l}\text { Majeure implicite } \\ \begin{array}{l}\text { Mineure (3): } \\ \text { présupposé négatif }\end{array}\end{array} & \text { All Americans are businessmen } & \text { Vérité incontestable } \\ \text { Conclusion (3) } & \text { So, you are not a real American } & \begin{array}{l}\text { Sentiment d'anormalité, } \\ \text { d'injustice et } \\ \text { d'aliénation: dissonance } \\ \text { cognitive }\end{array}\end{array}$


Be a real American by buying shares on the New York Stock Exchange
Sortie de la dissonance cognitive grâce au sens boursier de share qui indique une solution précise et simple (buy shares on the NYSE)

Dans le Tableau 2.3., la mineure a un présupposé négatif et share prend son sens boursier (une action). Dans ce cas, la conclusion (3) est qu'un Américain qui ne possède aucune action en bourse n'est pas un véritable Américain, ce qui suscite, comme en 2.2., un sentiment de dissonance cognitive chez le sujet. En revanche, grâce au sens boursier de share, la solution (3) est claire et précise. Elle propose une sortie de dissonance facilement réalisable: il suffit d'acheter des actions au New York Stock Exchange. La solution (3) apparaît comme la meilleure pour résoudre toutes les contradictions suggérées par le slogan. Cette dernière démonstration constitue le point d'aboutissement final de l'interprétation de la logique implicite du message par l'allocutaire: pour être un véritable Américain, il faut investir en bourse.

\section{Remonter de la logique de l'allocutaire à celle du locuteur}

Toutes les démonstrations proposées dans les tableaux 2.1., 2.2., 2.3. reposent sur un moule logique commun. À travers trois phases démonstratives successives, ce moule logique mène de la majeure All Americans are businessmen à la conclusion (3) Be a real American by buying shares on the New York Stock Exchange. Ce cadre de pensée est bien adapté à la grande majorité des Américains qui manifestent généralement un sens prononcé pour les affaires, mais qui n'investissent pas tous en bourse pour autant. Pourtant, nous formulons l'hypothèse que ce moule logique des allocutaires est inversé par rapport au moule logique initial des inventeurs du message, les promoteurs du NYSE. Pour eux, la majeure est sans doute America is a nation of shareholders, et leur moule logique sous-jacent décline probablement les conclusions successives comme suit (Tableau 3):

Tableau 3: Logique implicite des promoteurs du NYSE

PHASES DE LA DÉMONSTRATION

Majeure

Mineure

Conclusion (a)

Conclusion (b)

Conclusion (c) (hypothétique)
CONTENUS IMPLICITES

America is a nation of sharebolders.

You own stock market shares.

So, you own a share of American business.

So, you are a real American.

So, you are not a communist 
L'hypothèse de la majeure America is a nation of sharebolders, qui est tout à l'avantage du NYSE, est partiellement confirmée par Your Share of American Business qui suggère que tout Américain est potentiellement détenteur d'une part/d'une action de capital américain. America is a nation of shareholders est également une expression populaire qui a commencé à se répandre à travers la société américaine à la fin des années 20 dans le ton de l'article de John Raskob Everybody Ought to be Rich publié en août 1929 (Geisst 2000 : p. 22) Quant à la conclusion hypothétique (c), elle ne peut être extraite du slogan lui-même car les supports linguistiques où elle pourrait s'ancrer n'existent pas. Néanmoins, il n'est pas absurde de supposer qu'elle constitue une arrière-pensée très vraisemblable des concepteurs de cette campagne promotionnelle lancée en pleine guerre froide. Le projet de transformer les travailleurs en capitalistes pour désamorcer leurs revendications sociales et politiques date des années 20. Pour de nombreux hommes d'affaires américains de la période, le fait de détenir des actions devait donner aux ouvriers une mentalité conservatrice et les immuniser contre les idées radicales (Traflet 2003 : p. 5). L'idée fut reprise après la deuxième guerre mondiale et, dans les années 1950-1960, de nombreuses entreprises cotées au NYSE fondèrent leurs propres campagnes sur le concept du patriotisme économique et sur l'opposition capitalisme/communisme. Par exemple, la société Con Ed diffusa une publicité qui déclarait qu'en tant que capitalistes, «tous les actionnaires américains seraient liquidés par les communistes». Une autre société, General Telephone, centra sa campagne de marketing sur le personnage fictif «Joe Citizen Capitalist» pour inciter le public à investir dans l'entreprise. Le texte de présentation du personnage en fait le destinataire idéal du slogan du NYSE qu'il semble mettre en pratique spontanément. La plupart des contenus implicites sont les mêmes dans les deux énoncés.

Foe is a fellow with a lot of faith in the things that make this nation great. Over a period of years, Joe has invested his savings in the stock of several major companies, among them General Telephone Company of the Southwest. In his way, Foe and thousands like bim express their confidence in private industry. (Traflet 2003 : p. 17)

Le message implicite transmis par «Joe Citizen Capitalist» et par le slogan du NYSE est le même: acheter des actions d'entreprises privées n'est pas une simple affaire d'investissement financier, c'est un acte de foi dans le système socio-économique américain de la libre-entreprise. Une éclairante observation du sociologue Seymour M. Lipset montre que cette logique de comportement s'enracine profondément dans le terreau civilisationnel américain: «Being an American [...] is an ideological commitment. It is not a matter of birth. Those who reject American values are unAmerican» (Lipset 1996 : p. 31). Dans les années 1950 profondément marquées par le maccarthysme, le contenu implicite de ces messages est 
limpide. Pour un citoyen américain, la meilleure façon et d'échapper à la «chasse aux sorcières» menée par le House Committee on UnAmerican Activities (HCUA), c'est d'afficher son «américanité» en achetant des actions. Le corollaire de cet engagement idéologique est que ceux qui n'en achètent pas refusent de souscrire au projet américain. Ils sont donc un-American, et peut-être bien communistes... La glorification de «Joe Citizen Capitalist» induit subtilement la culpabilité de ceux qui ne lui ressemblent pas.

Quoi qu'il en soit, le slogan «Own Your Share...» contribua efficacement à l'essor de l'investissement boursier aux États-Unis. En grande partie grâce à lui, la campagne promotionnelle du NYSE parvint à attirer des millions d'Américains vers Wall Street. En 1952, le pays comptait environ six millions d'actionnaires (soit à peu près $4 \%$ de la population) tandis qu'en 1965, ils étaient plus de vingt millions (10,4\% de la population) (Traflet 2003 : p. 20).

Catherine Kerbrat-Orechionni se demande pourquoi les locuteurs usent de l'implicite alors que sa mise en œuvre et son interprétation exigent tant de soin et de temps. L'explicite n'est-il pas plus économique d'emploi et plus efficace dans ses effets? (op. cit. 274) Parmi les raisons qui justifient le recours à l'implicite, elle cite le respect des convenances et les stratégies de manipulation. Ces deux raisons s'appliquent parfaitement aux deux campagnes promotionnelles que nous venons d'étudier.

Dans la première, la critique des tentatives de réglementation du NYSE par le pouvoir fédéral ne pouvait être exprimée explicitement. En jouant sur le prestige politique considérable du mot people, le slogan «The NYSE is the Free and Open People's Market» suggère donc fortement que la Bourse de New York est une institution pleinement démocratique. L'implicite permet d'observer les convenances qui consistent aux Etats-Unis à respecter le pouvoir politique démocratiquement élu. L'utilisation de people dans le slogan est également manipulatoire car, comme nous avons essayé de le démontrer, people's market ne fait l'objet d'aucune définition technique précise contrairement à free et à open. Le mot n'est placé dans le slogan que parce qu'il évoque incontestablement l'idée de démocratie et que personne ne peut trouver à redire au projet d'un marché au service du peuple, même si, dans la réalité, le NYSE est encore très loin de cet idéal.

Dans la seconde campagne, le slogan «Own Your Share of American Business» est également une illustration du pouvoir manipulatoire de l'implicite. Le message sous-entendu donne à chaque Américain son dû 
(your share), puis le lui reprend, pour enfin le lui rendre à condition qu'il achète des titres boursiers. Les contenus implicites jouent sur les mots (share-part/share-action) et sur les contradictions de la langue (own/your) pour modeler l'idée que les allocutaires se font d'être américain. Le message joue également sur les valeurs nationales et patriotiques pour susciter chez l'allocutaire des sentiments contradictoires de fierté et de culpabilité. Le citoyen qui rentre dans son droit légitime d'Américain en achetant des valeurs boursières peut être fier de lui-même. Celui qui n'affirme pas son «américanité» en refusant d'acheter des titres n'a pas de quoi se vanter.

La leçon générale de cette étude de l'implicite dans les slogans de la Bourse de New York reste cependant qu'en anglais de spécialité, le décodage des énoncés ne peut se limiter à l'analyse des éléments de langue. Les apports du contexte professionnel et historique sont indispensables pour guider, enrichir et valider l'analyse linguistique. Dans toute la mesure du possible, le décodeur doit partager avec les destinataires de l'énoncé la même compétence encyclopédique que celle qui leur est nécessaire pour interpréter les différents niveaux de signification du message. Pour bien saisir l'implicite d'un énoncé, la culture semble être encore plus importante que pour en comprendre le contenu explicite.

\section{Bibliographie}

Adamczewski H. et J.-P. Gabilan, Les clés de la grammaire anglaise, Paris, Armand Collin, 1992.

Brandeis L., Other People's Money and How the Bankers Use It, New York, Bedford Books, 1995 [1914].

Cohen J.M. \& M.J. Cohen, The New Penguin Dictionary of Quotations, Londres, Penguin, 1992.

Galbraith J., The Great Crash, 1929, Boston, MA, Houghton Mifflin, 1961 [1954].

Geisst, C., 100 Years of Wall Street, New York, McGraw-Hill, 2000.

Grund F., "To Americans, Business is Everything", American Culture: An Anthology of Civilization Texts, Breidling Anders et al. éd., London, Routledge, 1996, p. 190-192.

Kahn O., The New York Stock Exchange and Public Opinion, New York, The New York Stock Exchange, 1917.

Kamm J., The Decentralization of Securities Exchanges, Boston, MA, Omeador, 1942.

Kerbrat-Orecchioni C., L'Implicite, Paris, Armand Colin, 1986.

Lipset, S. American Exceptionalism: A Double-Edged Sword, New York, W.W. Norton, 1996.

Medbury J., Men and Mysteries of Wall Street, Wells, VT, Fraser Publishing, 1968 [1870].

Meeker J., The Work of the Stock Exchange, New York, Ronald Press, 1930.

Ott J., 'The 'Free and Open' 'People's Market' Public Relations at the New York 
Stock Exchange", 1913-1929, Business and Economic History On-Line. Business History Conference, 2004.

< http://www.thebhc.org/publications/BEHonline/2004/Ott.pdf >

Parker J., Unmasking Wall Street, Boston, MA, Stratford, 1932.

Pecora F., Wall Street Under Oath, New York, Simon \& Schuster, 1939.

Shiller, R., Human Behavior and the Efficiency of the Financial System, New Haven, CT, Yale University-Cowles Foundation, Paper No. 1025, 2001, p. 1306-1340.

Stedman E., The New York Stock Exchange, New York, Greenwood Press, 1905.

Sparling E., Mystery Men of Wall Street, New York, Blue Ribbon Books, 1930.

Thomson A. \& A. Martinet, A Practical English Grammar, Oxford, OUP, 1981.

Traflet J., " 'Own Your Share of American Business' Public Relations at the NYSE during the Cold War." Business and Economic History On-Line. Business History Conference, 2003.

< http://www.thebhc.org/publications/BEHonline/2003/Traflet.pdf >

\section{Filmographie}

The Nation's Marketplace. Film documentaire produit et distribué par le Committee on Publicity du New York Stock Exchange, 1928. Une présentation détaillée illustrée de quatorze photos extraites du film est fournie par Julia Ott (2004 : p. 33-42). 